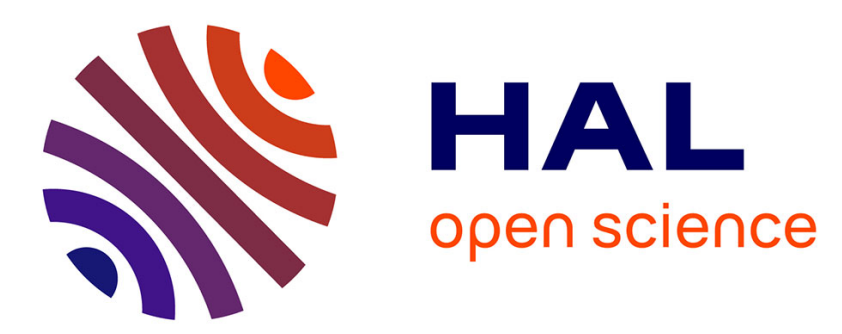

\title{
In situ ptychographic measurements of high-order harmonic sources from plasma mirrors: A theoretical and numerical study
}

A. Leblanc, F. Quéré

\section{- To cite this version:}

A. Leblanc, F. Quéré. In situ ptychographic measurements of high-order harmonic sources from plasma mirrors: A theoretical and numerical study. Physics of Plasmas, 2018, 25 (1), pp.013112. $10.1063 / 1.5002523$. cea-02277465

HAL Id: cea-02277465

https://hal-cea.archives-ouvertes.fr/cea-02277465

Submitted on 3 Sep 2019

HAL is a multi-disciplinary open access archive for the deposit and dissemination of scientific research documents, whether they are published or not. The documents may come from teaching and research institutions in France or abroad, or from public or private research centers.
L'archive ouverte pluridisciplinaire HAL, est destinée au dépôt et à la diffusion de documents scientifiques de niveau recherche, publiés ou non, émanant des établissements d'enseignement et de recherche français ou étrangers, des laboratoires publics ou privés. 


\section{In situ ptychographic measurements of high-order harmonic sources from plasma mirrors: A theoretical and numerical study}

Cite as: Phys. Plasmas 25, 013112 (2018); https://doi.org/10.1063/1.5002523

Submitted: 30 August 2017 . Accepted: 14 December 2017 . Published Online: 17 January 2018

A. Leblanc, and F. Quéré
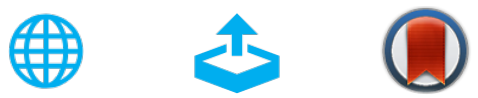

View Online

\section{ARTICLES YOU MAY BE INTERESTED IN}

Theory of relativistic radiation reflection from plasmas

Physics of Plasmas 25, 013108 (2018); https://doi.org/10.1063/1.5000785

Probing ultrafast dynamics of solid-density plasma generated by high-contrast intense laser pulses

Physics of Plasmas 25, 013102 (2018); https://doi.org/10.1063/1.5005176

Short-pulse laser harmonics from oscillating plasma surfaces driven at relativistic intensity

Physics of Plasmas 3, 3425 (1996); https://doi.org/10.1063/1.871619

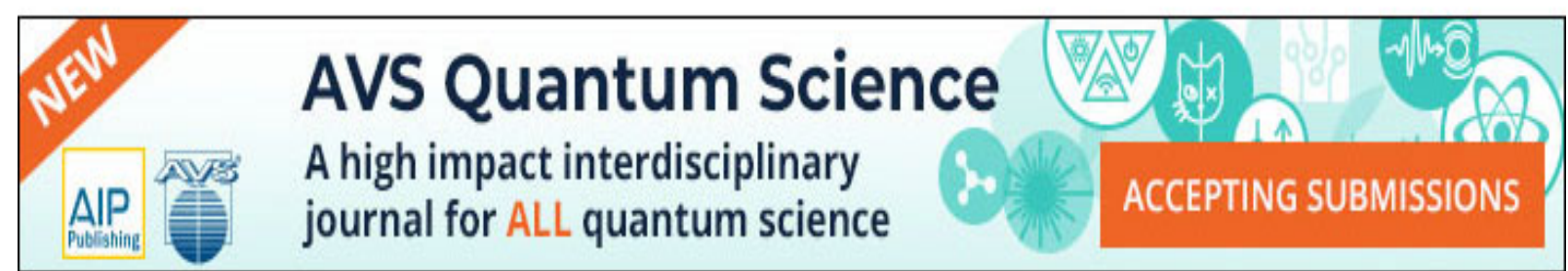




\title{
In situ ptychographic measurements of high-order harmonic sources from plasma mirrors: A theoretical and numerical study
}

\author{
A. Leblanc and F. Quéré \\ LIDYL, CEA, CNRS, Université Paris-Saclay, CEA Saclay, 91191 Gif-sur-Yvette, France
}

(Received 30 August 2017; accepted 14 December 2017; published online 17 January 2018)

\begin{abstract}
Measuring the spatial properties of high-order harmonic beams produced by high-intensity lasermatter interactions directly in the target plane is very challenging due to the extreme physical conditions at stake in the interaction area. A measurement scheme has been recently developed to obtain this information experimentally, which consists in adapting a lensless imaging method known as ptychography. In this paper, we present a theoretical validation of this method in the case of harmonic generation from plasma mirrors, using a combination of simple modeling and 2D Particle-In-Cell simulations. This study investigates the concept of in situ ptychography and supports the analysis of experimental measurements presented in previous publications. Published by AIP Publishing. https://doi.org/10.1063/1.5002523
\end{abstract}

\section{INTRODUCTION}

When a flat solid target is illuminated by an ultraintense $\left(I>10^{16} \mathrm{~W} / \mathrm{cm}^{2}\right)$ femtosecond laser, this target gets strongly ionized in a very short time. As long as the expansion of this plasma layer into the vacuum at the target surface remains short compared to the laser wavelength $\lambda_{L}$, it specularly reflects the laser pulse just as usual mirrors do: this is a plasma mirror. ${ }^{1}$ If the laser intensity is higher than $10^{18} \mathrm{~W} / \mathrm{cm}^{2}$, the electronic density oscillates with the incident laser field at relativistic speed along the target normal. In this Relativistic Oscillating Mirror (ROM) regime, ${ }^{2}$ the reflected laser beam is periodically distorted through the Doppler effect, resulting in the generation of trains of attosecond pulses, associated in the frequency domain to highorder harmonics of the laser frequency $\omega_{L}{ }^{4}$, potentially up to very high frequencies. ${ }^{3}$

For further applications, such as probing ultra-fast processes in matter by pump-probe experiments, ${ }^{5}$ it is of prime importance to control the divergence of those harmonic beams. Noting $E_{n}(\mathbf{r})=\left|E_{n}(\mathbf{r})\right| \exp i \varphi_{n}(\mathbf{r})$ the spatial field of the $n^{\text {th }}$ harmonic order in the target surface plane, the angular intensity of the harmonic beam is directly related to the square of the Fourier transform $\tilde{E}_{n}(\mathbf{k})$ of this field, $I_{n}(\mathbf{k})=\left|\tilde{E}_{n}(\mathbf{k})\right|^{2}$, with $\mathbf{k}$ the transverse wave vector. As a consequence, the harmonic beam divergence $\Delta \theta_{n}$, defined as the angular full width at half maximum (FWHM) in the intensity of the diffracted beam, is determined by two main properties of the source in the focal plane: its spatial extent-through the amplitude $\left|E_{n}(\mathbf{r})\right|$-and the spatial curvature of its wavefront-through the phase $\varphi_{n}(\mathbf{r})$. Physically, those properties are determined by the size of the laser focal spot on the target and the dependence of the harmonic amplitude and phase on laser intensity.

Experimentally, due to the extreme physical conditions in the interaction region, the only quantity that can be accessed in a rather direct manner is the angular intensity profile $I_{n}(\mathbf{k})$ measured far away from the target, i.e., at a distance $D$ large compared to the Rayleigh length of the focused laser beam. This however only provides partial information on the spatial properties of the harmonic source in the target plane, as one given that angular profile $I_{n}(\mathbf{k})$ can result from multiple combinations of source amplitude and phase profiles.

Removing this indeterminacy requires measuring the harmonic spatial wavefront in the same plane, which is also possible using, for instance, a short-wavelength ShackHartmann wavefront sensor. ${ }^{6,7}$ The complex field in the target plane can then in principle be determined by numerically back-propagating the field obtained in the measurement plane. However, getting a reliable result from such a calculation requires a very high accuracy on the propagation distance $D$, much better than the Rayleigh length $z_{r}^{n}$ of the $n$th harmonic (i.e., typically less than $100 \mu \mathrm{m}$ in experiments), which makes hardly exploitable in practice. Applying conventional measurement methods to determine the spatial properties of the harmonic beam in the target plane is thus extremely challenging.

These properties have nonetheless been recently measured by adapting a coherent diffraction imaging method called ptychography. ${ }^{8}$ Ptychography consists in measuring a set of diffraction patterns $S\left(\mathbf{k}, \mathbf{r}_{0}\right)$ of a probe beam $P(\mathbf{r})$ onto an object described by a complex transmission or reflection function $O(\mathbf{r})$, for multiple relative positions $\mathbf{r}_{0}$ between the beam and the object. The ptychographic dataset $S\left(\mathbf{k}, \mathbf{r}_{0}\right)$ is mathematically described by

$$
S\left(\mathbf{k}, \mathbf{r}_{0}\right)=\left|\iint P\left(\mathbf{r}-\mathbf{r}_{0}\right) O(\mathbf{r}) e^{i \mathbf{k} \cdot \mathbf{r}} d^{2} \mathbf{r}\right|^{2} .
$$

For sufficient sampling in $\mathbf{r}_{0}$, phase retrieval algorithms can be used to reconstruct the object $O(\mathbf{r})$, as well as the probe beam $P(\mathbf{r})$ in the object plane, both in amplitude and phase. $^{9-11}$

In order to adapt this scheme to harmonics generated by the non-linear interaction of an intense laser with a generation medium, the key idea is to spatially structure this generation medium, so that it acts as the object $O(\mathbf{r})$. The main 
difference with standard ptychography is that the probe beam does not illuminate the object but is now directly generated onto this object: we call this scheme in situ ptychography. In the case of harmonic generation from plasma mirrors, what is required is a spatial structuring of the plasma surface along the target plane.

This structuring can in principle be obtained by using solid targets with initial surface structures, such as gratings. However, given the significant pointing jitter of high-power lasers, scanning the relative position $\mathbf{r}_{0}$ between the laser focus and the target surface structure with sufficient control and accuracy would be very challenging. Moreover, in practice, this approach provides only very limited control on the parameters of the target structure.

A more efficient implementation of this measurement method consists in optically inducing a spatial structure on an initially flat target surface, using the so-called transient plasma gratings (TPGs). ${ }^{12}$ This is achieved by ionizing the target with a prepulse beam whose fluence is spatially modulated, hence leading to a spatial modulation of the plasma expansion velocity into the vacuum. This modulated expansion in turn leads to a modulation of the position of the critical plasma density $n_{c}$ for the laser frequency, which can serve as the diffracting object for the ptychographic measurement of the harmonic beam. The depth, spatial period, and lateral position $\mathbf{r}_{0}$ of this surface modulation can all be controlled optically, thus greatly facilitating the implementation of the method. In addition, as the structuring prepulse beam and the main beam driving harmonic generation typically originate from the same laser source, they both experience the same pointing fluctuations, and the laser pointing jitter is no longer an issue.

In this paper, we assess the validity and accuracy of this advanced measurement scheme, by performing a theoretical study based on simple modeling and Particle-In-Cell (PIC) simulations. As shown further, the present case is not a strict ptychographic measurement. It is important to prove that this in situ ptychographic method can be applied to the use of TPGs by discussing the robustness of the reconstruction procedure.

The main assumption of in situ ptychography compared to standard one is related to the fact that the probe beam (i.e., the harmonic beam here) is not reflected on the structured object but directly generated on its surface by the interaction of a driving laser with this object. In these conditions, the fact that the angular intensity profile of the harmonic beam can be expressed under the form of Eq. (1), and that the concepts and algorithms of ptychography can then be applied, is not obvious and needs to be verified, whether structured solid targets or TPGs are used. One should note that this hypothesis was assumed in previous experimental works ${ }^{8,18}$ which aim at analyzing the physics of the laser-plasma interaction. In the present paper, this main difference with standard ptychography is fully investigated for the first time.

Furthermore, the central hypothesis of general ptychography is that the object function $O(\mathbf{r})$ and the probe function $P(\mathbf{r})$ must be independent of their relative position $\mathbf{r}_{0}{ }^{9}$ However, in the specific case of TPG, the modulation of the plasma expansion velocity leads to a modulation not only of

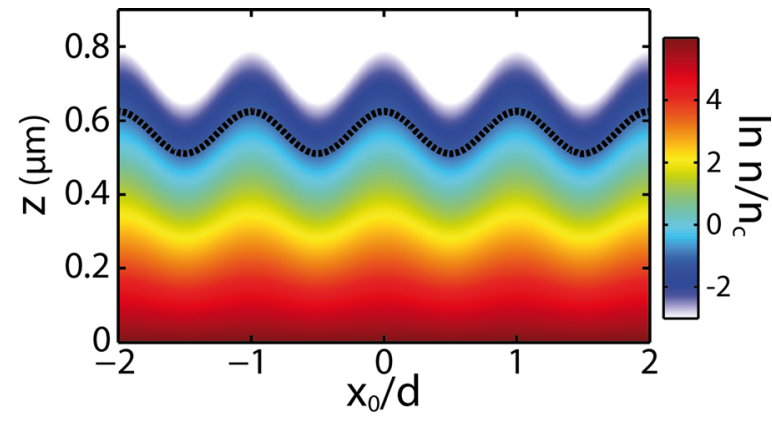

FIG. 1. Spatial density profile of a TPG_-plasma density around the target surface, as given by Eqs. (2) and (3) with $L_{0}=\lambda_{L} / 10$ and $\delta L / L_{0}=20 \%$. The isodensity surface $z_{r}(x)$ corresponding to the density $n_{r}=n_{c} \cos ^{2} \theta$ is plotted as a dashed line.

the critical density surface but also of the steepness of the density gradient at the plasma surface [see Fig. 1 in Sec. II]. This steepness is expressed by the spatial scale length $L$ of the density gradient. Previous experimental and theoretical studies ${ }^{13,14}$ have shown that the spatial properties of the harmonic beam in the source plane (extent of $\left|E_{n}(\mathbf{r})\right|$, curvature of $\varphi_{n}(\mathbf{r})$ ) depend on this parameter $L$. As a result, when generating harmonics on a TPG, the harmonic source properties actually vary with the relative position $\mathbf{r}_{0}$ of the driving laser along the target surface. Mathematically speaking, one then needs to replace $P\left(\mathbf{r}-\mathbf{r}_{0}\right)$ in Eq. (1) by $P\left(\mathbf{r}-\mathbf{r}_{0}, L(\mathbf{r})\right)=P\left(\mathbf{r}, \mathbf{r}_{0}\right)$. This violates the central hypothesis of ptychography.

It is of main concern to understand how the retrieval procedure behaves in this particular case in order to validate the performed measurements and evaluate the error that this hypothesis violation implies on the reconstructions.

The outline of this paper is given as follows: First, in Sec. II, we describe a high harmonic generation (HHG) process from plasma mirrors: the most relevant physical parameters of the laser-plasma interaction are presented, and the structure of TPG is specified. We then discuss in Sec. III the spatial properties of the harmonic beam in the target plane and their relationship with the diffracted angular profile of this beam.

Section IV uses a simple model to analyze the effect on the harmonic source of a modulated position of the target surface and shows how the measured dataset encodes information on this source. Applying a phase retrieval algorithm ${ }^{8,17}$ to the dataset calculated with this model proves that the standard ptychographic procedure can be applied to in situ ptychography, even if the probe is generated on the object instead of illuminating it.

All remaining sections consider the case of real TPG. In Sec. V, the variation of the harmonic source profile $E_{n}(\mathbf{r})$ with its position $\mathbf{r}_{0}$ on a TPG is calculated theoretically, using an analytical model for the spatial phase and PIC numerical simulations for the spatial amplitude. It enables us to estimate how far the real measurement is from standard ptychography described by Eq. (1). Finally, in Sec. VI, a ptychographic dataset for harmonics generated on a TPG is obtained from PIC simulations and processed with the phase retrieval algorithm. As the harmonic beam spatial properties in the target plane can be directly extracted from PIC 
simulations, we can then assess the validity of the harmonic source reconstructed by the ptychographic approach. The robustness of the method is demonstrated by a parametric study, where ptychographic measurements are simulated for different conditions of laser-plasma interaction. Sec. VII provides a general conclusion.

\section{PLASMA MIRRORS: INTERACTION CONDITIONS AND STRUCTURING}

We briefly review the main physical parameters of the laser-plasma mirror interaction, how they are experimentally controlled, and their consequences on the plasma structuring.

The two main physical parameters which determine the coupling between laser and plasma are the laser intensity and the steepness of the plasma density gradient at the target surface. Experimentally, the laser intensity is simply controlled through an attenuator consisting of a half-waveplate followed by two reflective polarizers and/or by aperturing the laser beam. The steepness of the plasma density gradient is controlled thanks to a pre-pulse: this prepulse ionizes the target and triggers plasma expansion a few hundred femtoseconds to a few picoseconds before the main pulse that drives HHG.

Experimentally, this prepulse is created by selecting a small part of the main laser beam, obtained by placing a small mirror in front of one of the large mirrors used to steer this beam to the target. ${ }^{12}$ This part of the beam is focused at a controlled delay before the main beam, typically in the piscosecond range. Its intensity at focus is about 3 orders of magnitude lower than that of the main beam. As the temporal contrast of the laser is extremely high (more than $10^{11}$ at $1 \mathrm{ps}$ ), the pre-ionization of the target is only due to this controlled pre-pulse.

The density profile along the target normal $z$ is exponential $^{15,16}$ with a gradient scale length $L$

$$
n(z)=n_{0} \exp -\frac{z}{L} .
$$

In this study, we consider a maximum density $n_{0} \simeq 400 n_{c}$ ( $n_{c}$ critical density at the laser frequency) which typically corresponds to the case of a fully ionized silica target.

$L$ varies linearly with the delay $\tau_{p p}$ between the prepulse and the main pulse, $L=v_{e} \tau_{p p}$, where the plasma expansion velocity $v_{e}$ depends on the pre-pulse intensity $I_{p p}$. In typical experimental conditions, $I_{p p} \sim 5 \times 10^{15} \mathrm{~W} / \mathrm{cm}^{2}$, leading to expansion velocities of the order of $L / \tau_{p p} \simeq 50 \mathrm{~nm} / \mathrm{ps}^{12}$ We can thus safely assume that the gradient scale length is constant during the interaction with the main pulse, which lasts only for $\tau_{L} \approx 25 f s$. In the case where a single pre-pulse is used, its focal spot is typically ten times larger than that of the main beam, so that the gradient scale length can be considered as constant across the main beam focal spot, $L(\mathbf{r})=L$. When a flat initial solid target is used, the plasma mirror can thus also be considered to be "flat."

The laser reflects around the density $n_{r}=n_{c} \cos ^{2} \theta$, where $\theta$ is the incident angle of the laser on the target. The in situ ptychographic scheme in reflection consists in inducing a variation of the position $z_{r}$ of this isodensity surface along the target, $z_{r}=z_{r}(\mathbf{r})$. As explained in the introduction, such a structuring of the plasma can be obtained by starting from a flat solid target and spatially modulating the pre-pulse intensity. Experimentally, an easy way to get such a modulation is to introduce a second pre-pulse which interferes spatially with the first one on the target surface. The angle between both prepulse beams implies a onedimensional intensity modulation of period $d$ along spatial coordinate $x$ in the target plane, which leads to a spatial modulation $z_{r}(x)$ of the critical density surface. As explained in the introduction, this typical structure is called a transient plasma grating (TPG). ${ }^{12}$

Another consequence of this modulated expansion is the fact that the gradient scale length $L$ in Eq. (2) also becomes a function of position $x$, which we write

$$
L(x)=L_{0}+0.5 \delta L \cos 2 \pi \frac{x}{d},
$$

where $L_{0}=\langle L\rangle_{x}$ is the average density gradient scale length along the target surface and $\delta L$ is the depth of the density gradient modulation. The spatial density profile of the plasma is given by Eq. (2), with $L=L(x)$ given by Eq. (3). An example of such a density profile is shown in Fig. 1. In this example, $\delta L / L_{0}=20 \%$, which is close to the conditions used for experimental ptychographic measurements performed so far. ${ }^{8}$ This is the typical configuration that we will consider for the following theoretical study.

Using this expression, we can deduce the isodensity surface for $n_{r}=n_{c} \cos \theta: z_{r}(x)=\ln \left(n_{0} / n_{r}\right) L(x)$. This isodensity surface is plotted as the black dashed line in Fig. 1. For $n_{0}$ $=400 n_{c}$ and $\theta=45^{\circ}$, this leads to $z_{r}(x) \approx 6.6 L(x)$. The two functions $z_{r}(x)$ and $L(x)$ thus have the same spatial periods, but the modulation amplitude of $z_{r}$ is significantly larger than that of $L$.

\section{RELATIVISTIC OSCILLATING MIRROR MECHANISM: PHASE PROPERTIES}

The ROM harmonic generation process is preponderant when the laser amplitude is higher than $a_{0} \approx 1$ and the gradient scale length is larger than $\sim \lambda_{L} / 20$, with a maximum generation efficiency at $L \approx \lambda_{L} / 10$ for $a_{0} \geqslant 1{ }^{13,14}$ In this part, we describe how the spatial phase $\varphi_{n}(\mathbf{r})$ of a ROM harmonic source generated on a flat plasma mirror depends on the interaction parameters $\left(a_{0}, L\right)$. This will help us to understand, in part IV, how this spatial phase varies along a TPG. To this end, we use an analytical model of this phase derived in Ref. 13, which has been confronted to PIC simulations and experiments and revealed very accurately. Note that there is no such predictive model yet for the amplitude profile $\left|E_{n}(\mathbf{r})\right|$ of the harmonic source, which can therefore only be studied numerically, using PIC simulations-see part IV.

The ROM spatial phase profile is determined by the plasma surface bending under the effect of the intense laser field. Indeed, at such high intensity, the plasma is pushed toward the target during the interaction. For a given laser amplitude $a$ and gradient scale length $L$, the total distance $z_{t}$ over which the plasma is pushed results from the 
combination of the displacements $z_{e}$ of the electronic density and $z_{i}$ of the ion density

$$
\begin{aligned}
z_{t} & =z_{e}+z_{i}, \\
z_{e} & =L \ln \left(1+\frac{2 \lambda_{L}(1+\sin \theta) a}{2 \pi L} \frac{n_{c}}{n_{e}\left(z_{i}\right)}\right), \\
z_{i}(t) & =2 L \ln \left(1+\frac{\Pi_{0}}{2 L \cos \theta} c \int_{-\infty}^{t} a\left(t^{\prime}\right) d t^{\prime}\right),
\end{aligned}
$$

where $\Pi_{0}=\sqrt{r Z m_{e} \cos \theta / 2 A m_{p}}$, with $r \simeq 0.8$ the plasma reflectivity for the laser, $Z$ and $A$ the average charge state and mass number of the ions, $m_{e}$ the electron mass, $m_{p}$ the proton mass, and $a(t) \propto \exp \left(-2 \ln 2 t^{2} / \tau_{L}^{2}\right)$ the temporal profile of the laser amplitude $\left(\tau_{L}\right.$ the temporal FWHM of the laser pulse). ${ }^{13}$

We consider that the spatial properties of the harmonic beam are mostly determined by those of the attosecond pulse of the highest intensity, which is generated when $a(t)$ is maximum. At this time, it can be shown ${ }^{18}$ that $z_{t}$ can be expressed as

$$
z_{t}(a, L)=L \ln \left(1+(\alpha+2 \beta) a+\beta^{2} a^{2}\right),
$$

with $\quad \alpha=\lambda_{L}(1+\sin \theta) / \pi L \quad$ and $\quad \beta=$ $\sqrt{\pi / 2 \ln 2} \Pi_{0} c \tau_{L} / 4 L \cos \theta$. For a flat plasma mirror, only the laser amplitude varies along the target surface

$$
a(\mathbf{r})=a_{0} \exp -\frac{\mathbf{r}^{2}}{w_{0}^{2}},
$$

with $w_{0}$ the laser waist. Due to this variation of $a_{0}, z_{t}$ is deeper at the center of the focal spot than on its edges. Therefore, the plasma is curved, and this curvature is directly imprinted on the spatial phase of the harmonic beam through

$$
\varphi_{n}(x)=-2 k_{n} z_{t}(x) \cos \theta
$$

for the $n$th harmonic order.

Figure 2 displays the spatial phase of the 12 th harmonic order, induced by this plasma curvature for three different interaction conditions $\left(a_{0}, L\right)$, as predicted by Eqs. (4)-(6). In this intensity range, the higher the laser amplitude is, or the longer the gradient scale length, the deeper the plasma is pushed into the target [Eq. (4)] and the more curved the harmonic spatial phase is [Eq. (6)].

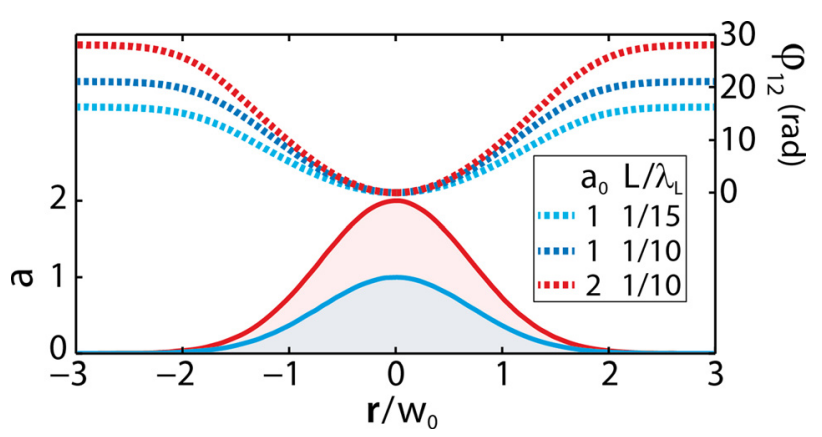

FIG. 2. Phase properties of a ROM harmonic source-spatial profiles of the laser amplitude (full lines) and resulting harmonic phase (12th harmonic order, dashed lines) generated on a flat plasma mirror, for different interaction conditions $\left(a_{0}, L / \lambda_{L}\right)$.

\section{IN SITU PTYCHOGRAPHY ON PLASMA MIRRORS}

This section reminds the principle of the measurement method, explains how the obtained dataset encodes information on the harmonic source properties, and tests the accuracy of the phase retrieval algorithm for datasets derived from a simple model, thus showing that this scheme can indeed be considered as a ptychographic measurement.

To this end, we analyze the case of a plasma created on an initially structured target surface, resulting in a modulated plasma surface, such that the position $z_{r}(x)$ of the critical density surface is modulated, while the gradient scale length $L$ is spatially homogeneous. Such a source clearly induces a spatial phase modulation on the reflected laser field. The key idea of the method is that this modulated plasma surface also acts as a spatial phase modulator on the harmonic source generated by this laser pulse. In other words, we consider the harmonic source $E_{n}(x)$ to be emitted from a varying position $z_{r}(x)$. The phase modulation $g_{n}(x)$ induced on the $n$th harmonic source field is written as

$$
g_{n}(x)=\exp \left[i 0.5 \delta \varphi_{n} \cos (2 \pi x / d)\right],
$$

with $\delta \varphi_{n}$ the depth of the phase modulation. In the experiment, the spectrometer measures the angular intensity profile of the diffracted harmonic beam, along one angular dimension and for each harmonic order. Using Fraunhofer diffraction, the measured signal is expressed as

$$
I_{n}\left(k_{x}, x_{0}\right)=\left|\int E_{n}(x) g_{n}\left(x-x_{0}\right) e^{i k_{x} x} d x\right|^{2},
$$

with $x_{0}$ the relative position of the grating and laser focus generating the field $E_{n}(x)$. Comparing this expression with Eq. (1) shows that this is the typical equation of one dimensional ptychography. The dataset $\left[I_{n}\left(k_{x}, x_{0}\right)\right]_{x_{0}}$ for all $x_{0}$ is named in this particular $1 \mathrm{D}$ case a ptychographic trace.

Figure 3 illustrates the effect of a sinusoidal phase modulation on the field of the 12th harmonic in the source plane [panels (b)-(e)] and on its diffracted intensity profile $I_{12}\left(k_{x}, x_{0}\right)$ [panels $(\mathrm{g})-(\mathrm{j})$ ], for different $x_{0}$. For this calculation, the harmonic source amplitude profile was assumed to be Gaussian, with a source size of $w_{12}=w_{0} / 2=3.1 \mu \mathrm{m}$ typical of experimental conditions. ${ }^{12}$ To calculate the source spatial phase for a flat plasma mirror [panel (a)], we use the analytical model of Sec. III, considering a plasma with a homogeneous gradient scale length of $L=\lambda_{L} / 15$, curved by a laser of maximum amplitude $a_{0}=1$. For the grating parameters, we used $d=20 \mu \mathrm{m}$ and $\delta \varphi_{12}=8.5 \mathrm{rad}$, which are again typical values from previous experiments.

A key point of this measurement regime is that the modulation period $d$ is significantly larger than the source size $w_{0}$. In these conditions, for values of $x_{0}$ such that the phase modulation is approximately quadratic, the phase grating adds its local curvature to the harmonic phase [Figs. 3(b) and $3(\mathrm{~d})$, resulting in a change in the beam divergence [panels (g) and (i)]. For the values of $x_{0}$ such that the phase modulation is quasi-linear [Figs. 3(c) and 3(e)], the local tilt of the grating changes the emission angle [panels (h) and (j)]. As shown in panel $(\mathrm{k})$, those two effects vary continuously with 


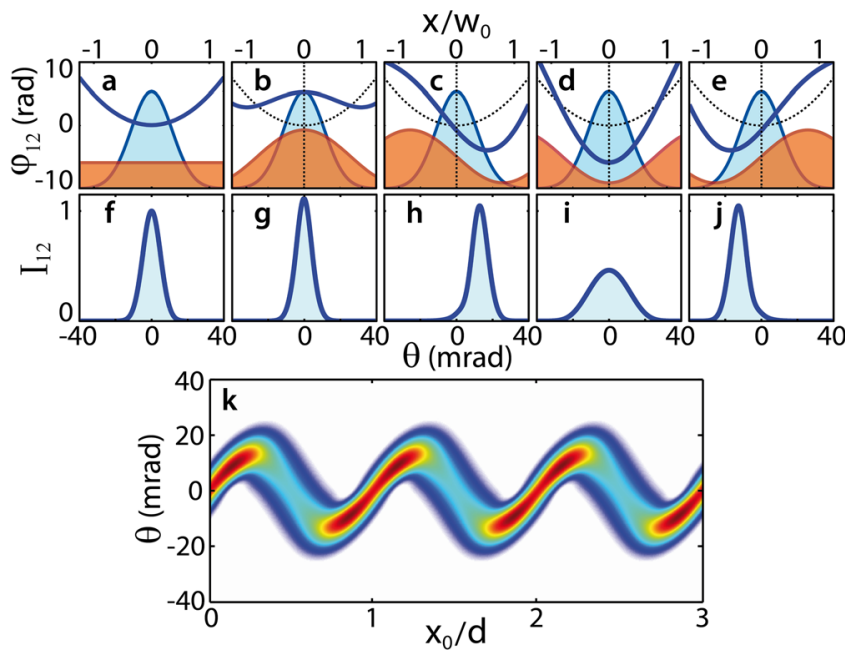

FIG. 3. Simulation of a ptychographic measurement on a plasma grating. Panel (a) shows the amplitude $\left|E_{12}(x)\right|$ and phase $\varphi_{12}(x)$ profiles, in the target plane, of the 12th harmonic generated on flat plasma mirror. The phase profile is derived from the model of Sec. III. Panel (f) shows the resulting intensity profile $I_{12}\left(\theta_{x}\right)$, with its peak value normalized to 1 . Panels (b)-(e): the amplitude and phase of the harmonic source in the presence of a plasma surface modulation for different relative positions $x_{0} / d=[0,0.25,0.5,0.75]$. The dashed line reminds $\varphi_{12}(x)$ for a flat plasma mirror [panel (a)], and the red lines corresponds to the phase modulation induced by the plasma grating and the blue line to the total phase resulting from the combination of these two terms. Panels $(\mathrm{g})-(\mathrm{j})$ : resulting diffracted intensity profile $I_{12}\left(\theta_{x}\right)$, with the same normalization as in panel (f). Panel (k): corresponding ptychographic trace $I_{12}\left(\theta, x_{0}\right)$ for the 12 th order harmonic, for $x_{0} / d \in[0,3]$.

$x_{0}$. The resulting ptychographic trace [panel $(\mathrm{k})$ ] closely resembles the ones that have been obtained experimentally in the ROM regime. ${ }^{8}$

The information on the harmonic source amplitude and phase is mostly encoded in the angular profiles measured at positions $x_{0}$ such that the phase modulation is quadratic. Indeed, for these positions, the larger the harmonic source size, the stronger the effect of the target curvature on the beam divergence. Moreover, if the harmonic source has a curved wavefront in the source plane, the grating curvature will either enhanced or compensate this curvature depending on their relative signs, thus either increasing or decreasing the harmonic beam divergence. Note that this encoding of the information in the ptychographic trace is totally analog to the encoding of the temporal structure of single attosecond pulses in FROG CRAB traces measured in the streaking regime. ${ }^{19}$

Figure 4 shows the trace reconstructed by applying the phase retrieval algorithm to the simulated trace shown in Fig. 3(k) and compares the two reconstructed functions $E_{12}(x)$ and $g_{12}\left(x_{0}\right)$ to the ones used as inputs in Eq. (8) to simulate the trace. The error on the reconstructed trace $I_{\text {rec }}$ [Fig. 4(a)], with respect to the simulated one $I_{\text {sim }}$ [Fig. 3(k)], is defined by ${ }^{11}$

$$
\epsilon_{I}=\sqrt{1-\frac{\left(\sum I_{\mathrm{sim}} \cdot I_{\mathrm{rec}}\right)^{2}}{\sum I_{\mathrm{sim}}^{2} \cdot \sum I_{\mathrm{rec}}^{2}}},
$$

where the sum $\sum$ is on $\theta$ and $x_{0}$. The errors on the reconstructed functions $f_{\text {rec }}$ with respect to those used to simulate the trace $f_{\text {sim }}$ are defined by
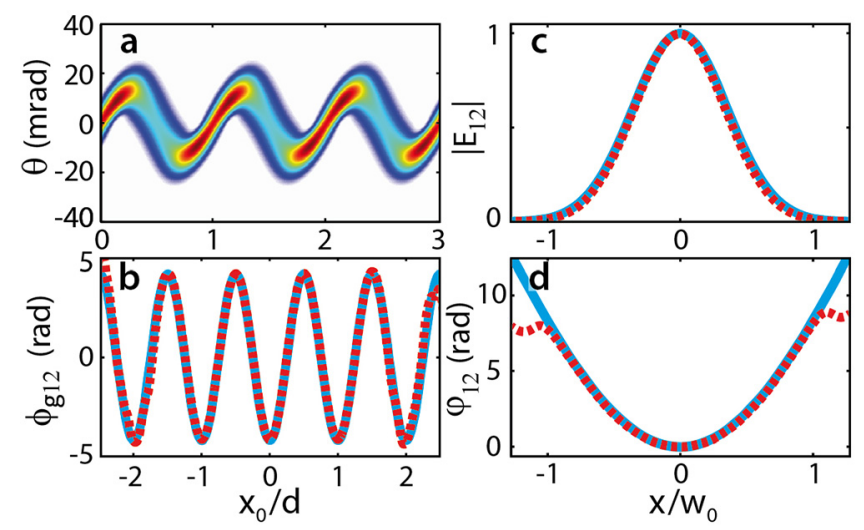

FIG. 4. Ptychographic reconstruction of the simulated trace-Panel (a) shows the trace reconstructed from the simulated trace displayed in Fig. 3(f). The reconstructed functions in the focal plane (red dashed lines) are compared to the simulated ones (blue full lines) for the phase grating $\left(\arg \left[g_{n}\left(x_{0}\right)\right)\right.$ in panel (b) and the harmonic source in amplitude $\left[\left|E_{n}(x)\right|\right.$, panel (c)] and phase $\left[\varphi_{n}(x)\right.$, panel (d)].

$$
\varepsilon_{f}=\sqrt{\frac{\sum\left(f_{\mathrm{sim}}-f_{\mathrm{rec}}\right)^{2}}{0.5 \cdot\left(\sum f_{\mathrm{sim}}^{2}+\sum f_{\mathrm{rec}}^{2}\right)}},
$$

where $f$ can either be the phase modulation induced by the grating $f=\arg \left[g_{n}\left(x_{0}\right)\right]$ or the source spatial profile in amplitude $\left(f=\left|E_{n}(x)\right|\right)$ or phase $\left(f=\varphi_{n}(x)\right)$. In the case of Fig. 4, these reconstruction errors, calculated on appropriate intervals [for instance for $x / w_{0} \in[-1,1]$ in panel (d)], are very low: $\epsilon_{I}=1.8 \%, \varepsilon_{g}=1.1 \%, \varepsilon_{|E|}=1.4 \%$, and $\varepsilon_{\varphi}=0.6 \%$.

In summary, from the dataset of intensity diffraction patterns $\left[I_{n}\left(k_{x}, x_{0}\right)\right]_{x_{0}}$, the phase retrieval algorithm reconstructs the grating and the harmonic source functions in amplitude and phase in the target plane with very good accuracy, leading to two conclusions. First, this means that this dataset contains all information necessary on the object and probe to reconstruct them and can thus be considered as a ptychographic measurement. Second, the phase retrieval algorithms that we use ${ }^{8}$ are appropriate.

\section{HARMONIC SOURCES PRODUCED ON TRANSIENT PLASMA GRATINGS}

We now turn to the case of measurements performed using transient plasma gratings, for which both the critical density surface $z_{r}(x)$ and the density gradient scale length are spatially modulated. As shown in Sec. II, the harmonic source properties depend on the plasma scale length. Therefore, a TPG not only acts as a phase grating, like a plasma created on a structured solid target does: at second order, the harmonic source is modified as $x_{0}$ is varied. As a consequence, the diffraction pattern dataset $I_{n}\left(k_{x}, x_{0}\right)$ is not exactly described by Eq. (8), where $E_{n}$ and $g_{n}$ are two independent functions. In this section, we study the dependence of the phase and amplitude of $E_{n}$ on $x_{0}$ for realistic physical parameters.

Figure 5 illustrates how the spatial phase profile of the 12th harmonic source is modified as $x_{0}$ is scanned along a $\mathrm{TPG}$, for $a_{0}=1.5$ and $L_{0}=\langle L\rangle_{x}=\lambda / 10 . \varphi_{12}(x)$ is calculated for different $x_{0}$ using Eqs. (4) and (6), taking into 


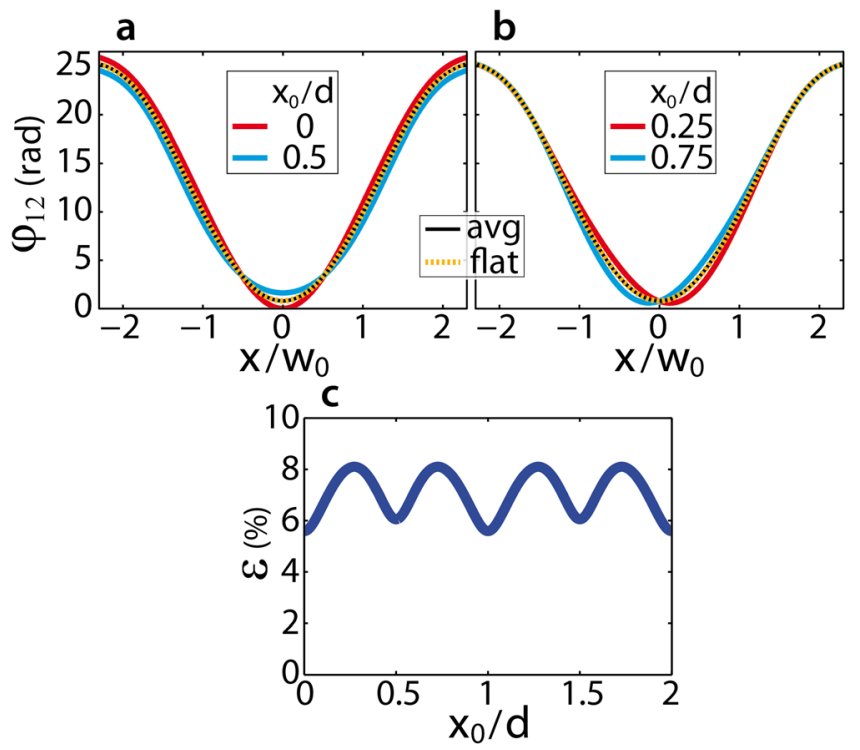

FIG. 5. Phase profile of the harmonic source for different positions $x_{0}$ of the main focal spot along the plasma grating-Simulated phase profiles of the 12th harmonic order generated on a TPG described by Eq. (3) with $L_{0}=$ $\lambda_{L} / 10$ and $\delta L / L_{0}=20 \%$, for different relative positions $x_{0}$ of the source on the grating: on a peak or a valley of the TPG on panel (a) and on slopes of the TPG on panel (b). The averaged profile for all $x_{0}$ is plotted as the orange dashed line on both panels, together with the phase profile of the source generated on a flat plasma mirror of scale length $L_{0}$ in the full black line. The normalized discrepancy $\epsilon$ [Eq. (10)] between the phase profile generated on the TPG and the one generated on the flat plasma mirror is plotted in panel (c) as a function of $x_{0}$.

account the spatial dependence of $a(x)$ and $L(x)$ through Eqs. (5) and (3). As the plasma is more strongly pushed inward when the gradient scale length is larger, if the source is generated on a peak $\left(x_{0} / d=0\right.$, larger $L$, see Fig. 1$)$ or on a valley $\left(x_{0} / d=0.5\right.$, smaller $\left.L\right)$ of the TPG, the phase curvature tends to be respectively increased or decreased compared to the case of a flat plasma mirror of gradient scale length $L_{0}$, as observed in Fig. 5(a). When the harmonic source is generated on a grating slope $\left(x_{0} / d=0.25\right.$ or 0.75 , see Fig. 1$)$, for the same reason, the plasma displacement is larger on one side of the laser focal spot than on the other, and the phase symmetry is slightly altered [see panel (b)].

For comparison, the black lines in panels (a) and (b) show the phase profile of the source generated on a flat plasma mirror of gradient scale length $L_{0}$. The difference between this phase profile and the one obtained on a TPG is quantified by Eq. (10), and this error function $\epsilon$ is plotted as a function of $x_{0}$ in panel (c). The highest values of $\epsilon$, about $8 \%$, are obtained when the source is generated on the grating slopes. The phase profile averaged on all $x_{0}$ is plotted as an orange dashed line on panels (a) and (b). It is extremely close to the one generated on the flat plasma mirror of average scale length $L_{0}$ (error $\varepsilon_{\varphi}<0.01 \%$ ).

As far as the harmonic beam amplitude is concerned, since no simple model is available at present to calculate it, it has to be evaluated by means of extensive computational simulations of the laser plasma interaction. The variation with $x_{0}$ of the amplitude profile of the harmonic source is deduced by 2D PIC simulations performed with the code EPOCH. We simulate a laser beam with a Gaussian spatial profile, focused with an incidence angle of $\theta=45^{\circ}$ on a plasma grating whose density is given by Eqs. (2) and (3) for $n / n_{c} \in[0.05,400]$. Using the numerical procedure described in Ref. 13, we obtain the field in time and space in the target plane, from which we can calculate the amplitude profile of each generated harmonic.

As we need to compare the amplitude profiles of the harmonic source obtained for different positions $x_{0}$ on the TPG, to limit the required computation time, the spatial resolution of the simulation box is fixed to $\lambda_{L} / 250$, with a corresponding time step of $T_{L} / 350$. One simulation typically requires 6000 computation hours on a supercomputer. The simulations were performed with $a_{0}=1.5, L_{0}=\lambda_{L} / 10, \delta L / L_{0}=20 \%$, for twenty different values of $x_{0}$ in the range $x_{0} / d \in[0,0.95]$.

The amplitude profiles obtained for the 12th harmonic are shown in Figs. 6(a) and 6(b). The averaged profile is plotted as the full black line on panel (b). These profiles are compared to this averaged one through Eq. (10). This deviation $\varepsilon$ is plotted on panel (c) and varies between $4 \%$ and $9.5 \%$. It is mostly due to variations of the harmonic beam profile occurring around the center of the focal spot, as we can see on panel (b). One additional PIC simulation is performed with the same laser conditions but now on a flat plasma mirror of scale length $L_{0}=\langle L\rangle_{x}$. The amplitude profile of the 12th harmonic order obtained in this case is shown as a red dashed line on panel (b). It differs from the profile averaged on all $x_{0}$ (black line) by only $\varepsilon=3.1 \%$.

In summary, we have evaluated how the harmonic source phase and amplitude profiles are modified as the position of the main laser focal spot is scanned along a TPG. Their maximum variations, which are smaller than $10 \%$, are representative of how far the measurement is from standard ptychography. Moreover, the profiles averaged along the plasma grating are very close to those generated on a flat plasma mirror with a scale length $L_{0}$ corresponding to the average scale length of the TPG. This implies that at first order, the plasma grating acts as a phase grating for the harmonic source resulting from an interaction on a flat plasma
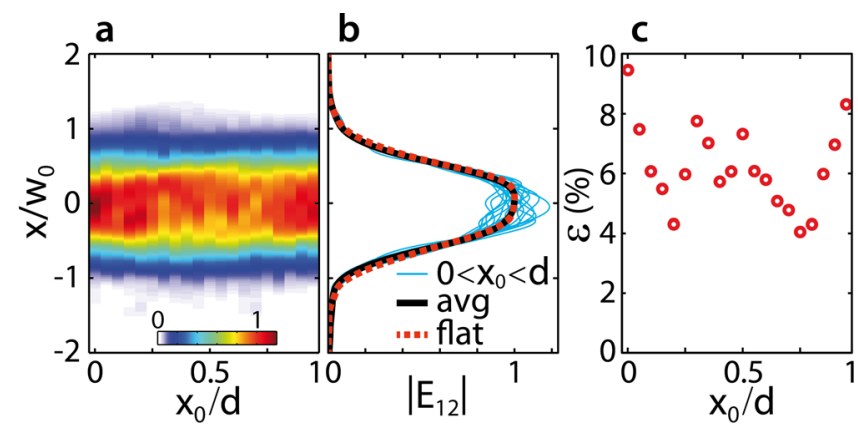

FIG. 6. Variation of the harmonic source amplitude profile along the plasma grating-Panels (a) and (b) display the amplitude profiles $\left|E_{12}\right|(x)$ of the 12th harmonic in the focal plane, obtained from PIC simulations, for 20 different positions $x_{0} / d \in[0,0.95]$ of the laser focal spot $\left(a_{0}=1.5\right)$ on the plasma grating $\left(L_{0}=\lambda_{L} / 10\right.$ and $\left.\delta L / L_{0}=20 \%\right)$. The full black line in panel (b) corresponds to the profile averaged on all $x_{0}$ and the red dashed line to the profile resulting from the interaction with a flat plasma mirror of scale length $L=L_{0}$, deduced from an additional PIC simulation. The variation with $x_{0}$ of the amplitude profile compared to the average profile is evaluated through Eq. (10) and plotted on panel (c). 
mirror. At second order, this harmonic source is slightly modified (less than $10 \%$ ) depending on the position $x_{0}$ on the grating due to the change in the local scale length.

\section{PTYCHOGRAPHIC RECONSTRUCTION}

We now address the reliability of the in situ ptychographic measurements of high order harmonics performed with TPG as the diffracting object.

From the PIC simulations presented in Sec. V, we can extract the harmonic diffraction pattern for each position $x_{0}$ of the laser focal spot on the TPG and thus obtain a simulated ptychographic trace. Such a trace is displayed in Fig. 7(a) for the 12th harmonic. It is qualitatively similar to the ptychographic trace of Fig. 4(a), which was calculated using the simple model for a pure phase grating. This supports the idea that the TPG indeed mostly acts as a phase object.

We have then processed this ptychographic trace with the same phase retrieval algorithm as the one used in Sec. IV. In order to avoid reconstruction ambiguities, which were more specifically observed when processing experimental traces, we imposed the diffracting object to be a pure phase function (amplitude fixed to 1). ${ }^{20}$ The obtained reconstructed trace is shown in Fig. 7(b) and corresponds to a reconstruction error of $\epsilon_{I}=12.4 \%$.

Panels (c) and (d) compare the amplitude and phase profiles of the retrieved harmonic source to the ones obtained from an additional PIC simulation for a flat plasma mirror of scale length $L_{0}=\langle L\rangle_{x}$. The difference is $\varepsilon_{\varphi}=7.6 \%$ for the phase profiles (restricting the comparison to the interval $x / w_{0} \in[-0.5,0.5]$ where phase profiles are meaningful), and $\varepsilon_{E}=5.9 \%$ for the amplitude profiles. This shows that in this physically realistic case, the reconstructed harmonic source obtained from the ptychographic measurement on a TPG is very close to the harmonic source generated on a flat

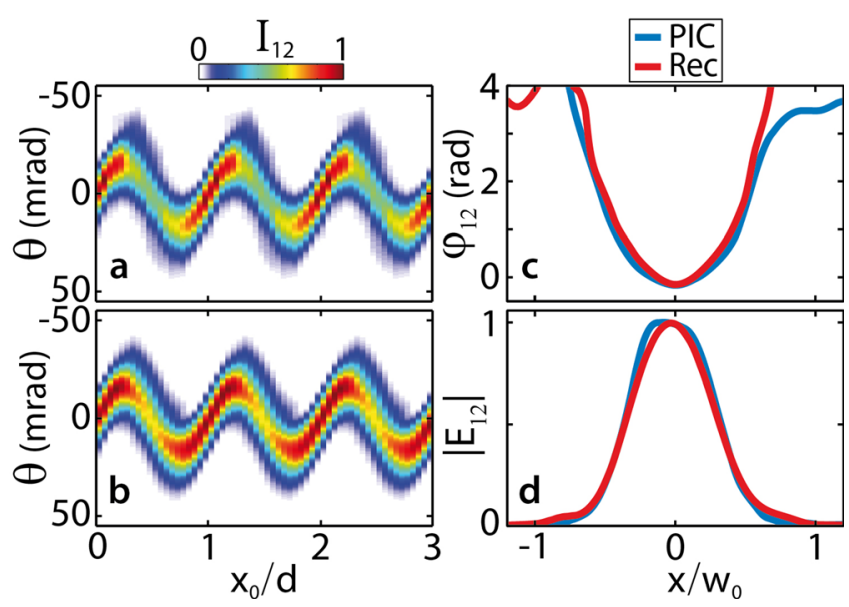

FIG. 7. Numerical validation of ptychographic measurements on TGPPanel (a) shows the ptychographic trace obtained by 20 PIC simulations for different positions $x_{0}$ of the main laser focal spot on a TPG. Panel (b) shows the reconstruction of this trace by the phase retrieval algorithm. The corresponding retrieved phase and amplitude profiles of the harmonic source are plotted on panels (c) and (d) (red lines) and compared to the profiles obtained from an additional PIC simulation on a flat plasma mirror of scale length $L_{0}$ (blue lines).
TABLE I. Different interaction conditions for PIC simulations of ptychographic measurements on TPG.

\begin{tabular}{lcc}
\hline \hline Conditions & $a_{0}$ & $L_{0}$ \\
\hline a & 1.5 & $\lambda / 20$ \\
b & 1.5 & $\lambda / 10$ \\
c & 1.5 & $\lambda / 6$ \\
d & 0.8 & $\lambda / 10$ \\
e & 3 & $\lambda / 10$ \\
\hline \hline
\end{tabular}

plasma mirror with the averaged TPG scale length. The fact that the harmonic source properties are slightly modulated as $x_{0}$ is scanned, as shown in Sec. $\mathrm{V}$, thus does not compromise the validity of the measurement.

To further test the validity of this ptychographic method, we have carried out a parametric study for different plasma scale lengths and laser amplitudes, listed in Table I. In each of these cases, like in the previous study, we have built a ptychographic trace by performing a set of PIC simulations for different $x_{0}$, applied the phase retrieval algorithm to the resulting trace, and compared the reconstructed source to the one generated on a flat plasma mirror of appropriate scale length. In all cases, $\delta L / L_{0}=20 \%$ for the TPG so that the plasma grating surface is more strongly modulated when $L_{0}$ is increased.

To limit the computation time, the resolution of the simulation box has been reduced compared to the previous simulations, and the parametric study is made on the 5th harmonic order. Figure 8 summarizes the outcome of this study: for the interaction conditions considered, the ptychographic measurement is reliable, with reconstruction errors $\varepsilon_{I} \lesssim 10 \%$ and differences between the retrieved and real source profiles of less than $10 \%$ in most cases.

Three main limits are however highlighted. First, for short plasma scale lengths, panel (a), the spatial variation of the harmonic phase is weak and falls below the method sensitivity, so that the retrieved phase is meaningless. Second, when the TPG is "deep" (long gradient scale length), panel (c), the source is still reconstructed with good accuracy, but the error on the reconstructed trace is higher (here, $\epsilon_{I} \simeq 16 \%$ ). This can be attributed to the fact that the amplitude modulation of the source along the grating is higher, whereas it is not taken into account in the reconstruction procedure. Finally, for high laser amplitudes, the reconstruction error gets higher, panel (e), probably because the variations of source amplitude and phase profiles along the grating get stronger. It is in this last case that the error on the retrieved harmonic properties is higher: the source size is slightly underestimated and the phase curvature overestimated.

In summary, this study shows that, for realistic physical conditions corresponding to our earlier experiments $\left(a_{0} \in[1.2,2]\right.$ and $\left.L_{0} \in\left[\lambda_{L} / 20, \lambda_{L} / 8\right]\right)$, in situ ptychographic measurements based on TPG as a diffracting object provide a reliable reconstruction of the harmonic source spatial properties. This retrieved harmonic source corresponds to the one generated on a flat plasma mirror whose scale length is equal to the averaged one along the TPG. 

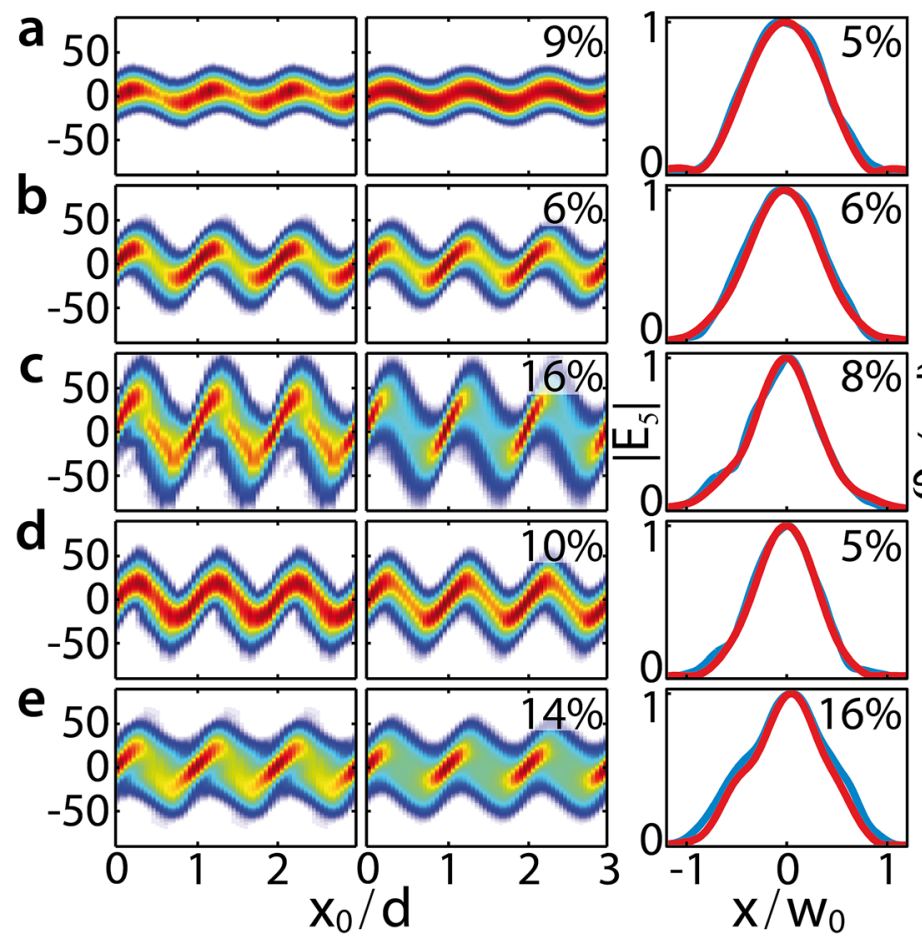

\section{CONCLUSION}

When an ultra-intense laser is reflected on a plasma mirror, the curvature of the plasma under radiation pressure is printed out on the spatial phase of the reflected beam and in particular on the phase profile of the high order harmonics generated on the surface, resulting in a converging XUV beam. Measuring the harmonic source amplitude and phase profile in the target plane is very challenging as the beam can only be measured far away from the interaction. A solution has been demonstrated ${ }^{8}$ which consists in adapting a coherent diffraction imaging method, named ptychography. The principle of ptychography is to diffract out a probe beam onto an object for different relative positions of one to the other. ${ }^{10}$ By the use of targets with spatially structured surfaces, it can be adapted to high harmonic generation on plasma mirrors into the so-called in situ ptychography where the probe beam is directly generated on the object.

In practice, the structuring can also be obtained by shaping the expansion of the plasma through the interference of two pre-pulse beams before the main interaction. This results in a plasma object called a transient plasma grating (TPG), which consists of a one dimensional modulation of the plasma density gradient scale length along the target surface. ${ }^{12}$ At first order, this results in a variation of the emitting position of the harmonic beam along the surface and the TPG acts as a phase object for this beam (part V). At second order, the source profile is modified with the source relative position on the grating, as the harmonic generation efficiency and the spatial phase curvature (part II) depend on the plasma scale length, which locally varies along the plasma object here. It implies that this scheme is not strictly a ptychographic measurement because this method assumes the probe beam and the object function to be independent. It is yet very important to understand how far the experiment is

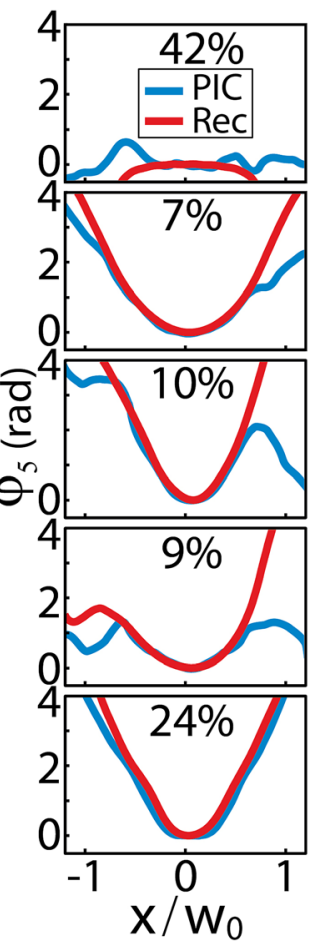

FIG. 8. Ptychographic reconstruction of traces obtained by PIC simulations for different interaction conditionsThe different lines of the figure correspond to the interaction conditions listed in Table I. The first two columns, respectively, show the PIC and reconstructed traces with the reconstruction error $\epsilon_{I}$. The two last columns show the retrieved source amplitude and phase profiles (red lines), together with the profiles obtained from a PIC simulation performed for a flat plasma mirror of scale length $L_{0}$ (blue lines). The differences between both, $\varepsilon_{E}$ and $\varepsilon_{\varphi}$, are indicated on the top right corner of the graphs.

from a standard ptychographic measurement and what the reconstructed harmonic source actually corresponds to.

The variations in the spatial phase and amplitude profiles of the XUV beam, evaluated by an analytical model or by PIC simulations, are about $\varepsilon \$ 8 \%$ (part IV), which is relatively low. Furthermore, the average source amplitude and phase profiles along the plasma grating are equal to those of the harmonic source generated on a flat plasma mirror whose scale length is equal to the averaged scale length of the grating $L_{0}=\langle L\rangle_{x}$. Finally, the ptychographic algorithm (part V) reconstructs source profiles which correspond to good accuracy to the ones generated on a flat plasma mirror of scale length $L_{0}$.

In conclusion, this theoretical study shows that for the experimental measurements performed with this technique, ${ }^{8}$ we can consider the reconstructed harmonic source profiles as the profiles resulting from an interaction on a flat plasma mirror whose scale length is the averaged one of the plasma grating. In these experiments, we found errors on reconstructions of the ptychographic traces of about $\epsilon_{I} \simeq 20 \%-25 \%$, which is typical of standard ptychography. ${ }^{11}$ The present study proves that a part of this error, about $10 \%-12 \%$, is intrinsically due to the measurement scheme. What is left is most likely due to measurement fluctuations.

\section{ACKNOWLEDGMENTS}

The research leading to these results has received funding from Agence Nationale pour la Recherche (project No. ANR-14-CE32-0011) and from the European Research Council under the European Union's Horizon 2020 research and innovation programme (ERC Grant Agreement No. 694596). This work was granted access to the HPC resources of CINES under the allocation 2017-A0020506057 made by GENCI. 
${ }^{1}$ C. Thaury, F. Quere, J. P. Geindre, A. Levy, T. Ceccotti, P. Monot, M. Bougeard, F. Reau, P. d'Oliveira, P. Audebert, R. Marjoribanks, and P. Martin, Nat. Phys. 3, 424 (2007).

${ }^{2}$ R. Lichters, J. Meyer-ter-Vehn, and A. Pukhov, Phys. Plasmas 3, 3425 (1996).

${ }^{3}$ B. Dromey, M. Zepf, A. Gopal, K. Lancaster, M. S. Wei, K. Krushelnick, M. Tatarakis, N. Vakakis, S. Moustaizis, R. Kodama, M. Tampo, C. Stoeckl, R. Clarke, H. Habara, D. Neely, S. Karsch, and P. Norreys, Nat. Phys. 2, 456 (2006).

${ }^{4}$ C. Thaury and F. Quéré, J. Phys. B 43(21), 213001 (2010).

${ }^{5}$ F. Krausz and M. Ivanov, Rev. Mod. Phys. 81(1), 163 (2009).

${ }^{6}$ P. Mercère, P. Zeitoun, M. Idir, S. Le Pape, D. Douillet, X. Levecq, G. Dovillaire, S. Bucourt, K. Goldberg, P. Naulleau, and S. Rekawa, Opt. Lett. 28, 1534 (2003).

${ }^{7}$ S. Künzel, G. O. Williams, W. Boutu, E. Galtier, B. Barbrel, H. J. Lee, B. Nagler, U. Zastrau, G. Dovillaire, R. W. Lee, H. Merdji, P. Zeitoun, and M. Fajardo, Appl. Opt. 54, 4745 (2015).

${ }^{8}$ A. Leblanc, S. Monchocé, C. Bourassin-Bouchet, S. Kahaly, and F. Quéré, Nat. Phys. 12(4), 301-305 (2016).

${ }^{9}$ J. Rodenburg, Phys. Rev. Lett. 98, 034801 (2007).
${ }^{10}$ P. Thibault, M. Dierolf, A. Menzel, O. Bunk, C. David, and F. Pfeiffer, Science 321, 379 (2008).

${ }^{11}$ P. Thibault, M. Dierolf, O. Bunk, A. Menzel, and F. Pfeiffer, Ultramicroscopy 109, 338 (2009).

${ }^{12}$ S. Monchocé, S. Kahaly, A. Leblanc, L. Videau, P. Combis, F. Réau, D. Garzella, P. D’Oliveira, P. Martin, and F. Quéré, Phys. Rev. Lett. 112, 145008 (2014).

${ }^{13}$ H. Vincenti, S. Monchocé, S. Kahaly, G. Bonnaud, P. Martin, and F. Quéré, Nat. Commun. 5, 3403 (2014).

${ }^{14}$ S. Kahaly, S. Monchocé, H. Vincenti, T. Dzelzainis, B. Dromey, M. Zepf, P. Martin, and F. Quéré, Phys. Rev. Lett. 110, 175001 (2013).

${ }^{15}$ Y. B. Zeldovich and Y. P. Raizer, Physics of Shock Waves and HighTemperature Hydrodynamic Phenomena (Dover Publications, 2002).

${ }^{16} \mathrm{M}$. Bocoum, M. Thévenet, F. Böhle, B. Beaurepaire, A. Vernier, A. Jullien, J. Faure, and R. Lopez-Martens, Opt. Lett. 40, 3009 (2015).

${ }^{17}$ J. R. Fienup, Appl. Opt. 21, 2758 (1982).

${ }^{18}$ A. Leblanc, S. Monchocé, H. Vincenti, S. Kahaly, J. L. Vay, and F. Quéré, Phys. Rev. Lett. 119, 155001 (2017).

${ }^{19}$ Y. Mairesse and F. Quéré, Phys. Rev. A 71, 011401 (2005).

${ }^{20}$ M. Guizar-Sicairos, Opt. Express 18, 18374 (2010). 